\title{
Transcranial magnetic stimulation studies of visuospatial attentional control
}

\author{
Sara M Szczepanski* and Sabine Kastner
}

\author{
Address: Department of Psychology, Princeton Neuroscience Institute, Green Hall, Princeton University, Princeton, NJ 08540, USA \\ * Corresponding author: Sara M Szczepanski (sszczepa@princeton.edu) \\ FI000 Biology Reports 2009, I:8I (doi: I0.34I0/BI-8I)
}

The electronic version of this article is the complete one and can be found at: http://FI000.com/Reports/Biology/content/I/8I

\begin{abstract}
Transcranial magnetic stimulation (TMS) is an established technique in cognitive neuroscience which is used to interrupt processing in the brain, creating a brief 'virtual lesion'. Here, we review recent studies that have employed TMS to gain insight into the roles of frontal and parietal cortex in visuospatial attention control.
\end{abstract}

\section{Introduction and context}

Transcranial magnetic stimulation (TMS) is a non-invasive technique that involves the application of rapidly changing current through a ferromagnetic coil placed over a subject's skull. As the electrical current through the coil varies in time, a brief magnetic field that penetrates the skin and skull is generated, inducing current flow in the brain region directly underneath the coil. The magnetic pulse briefly alters neural activity, for approximately $30-100 \mathrm{~ms}[1,2]$, presumably by causing a discharge of neurons to a depth of 1.5-2.0 cm below the scalp [3]. Depending on the strength of the pulse, the particular brain region that is stimulated, and coil orientation, TMS can either facilitate or interrupt processing, creating a 'virtual lesion' in the latter case. As a consequence, it is possible to alter behavioral performance of a particular task if the TMS pulse is applied over a brain area that is of critical functional importance for that task. TMS is widely used in cognitive neuroscience since it is the only method that has the potential to establish a causal relationship between brain and behavior in healthy subjects, with the immediacy, non-invasiveness, control, and relatively high spatial and temporal resolution that typically only correlative methods such as functional magnetic resonance imaging (fMRI) or electroencephalography (EEG) offer.

Although TMS has been used successfully to study cognitive functions in humans for more than two decades, relatively little is known about its direct effects at the neural level. The types of neurons activated by a TMS pulse depend on two factors: the direction of the electric current, which changes depending on the shape and orientation of the coil and the underlying brain anatomy, and the intensity level used [4]. The majority of studies investigating this topic have recorded the responses of pyramidal tract neurons following TMS over motor cortex in both monkeys $[5,6]$ and humans [7]. Therefore, there is plenty of evidence that TMS increases spiking activity in pyramidal neurons. It is unknown, however, whether this effect is specific to the motor system or whether it generalizes to other brain systems. To our knowledge, there is only indirect evidence that TMS also affects excitatory and inhibitory interneurons [8]. More studies, such as experiments using concurrent TMS and single-cell physiology, are needed in order to elucidate the exact neural mechanisms underlying the effects of TMS.

Two different types of TMS are routinely used to probe cognitive functions: single-pulse TMS, which discharges only one pulse per second or less and is most useful for examining temporal aspects of function, and repetitivepulse TMS (rTMS), during which a train of pulses over a period of time is delivered to a brain region. rTMS is most useful when the exact timing of a cognitive event is unknown or when scientists wish to evoke lasting 
plasticity of brain function to influence behavior such as in the treatment of major depression [9].

TMS has been used to study a variety of cognitive functions, including visual perception, language, and memory. This review will focus on the effects of TMS on attentional control. Neuroimaging studies in healthy human subjects have identified a network consisting of areas in the superior parietal lobule (SPL), intraparietal sulcus (IPS), frontal eye field (FEF), and supplementary eye field (SEF), which is consistently activated by a variety of visuospatial, feature-based, and object-based tasks that require the allocation of attention to select information [10-12] (Figure 1). However, the correlative nature of fMRI limits the conclusions that can be drawn about the exact functional roles played by these frontoparietal areas in attentional control. Although we may have identified many of the key areas that are responsible for controlling visual attention in the human brain, we have barely begun to characterize each area's individual contribution to attentional control. In this context, TMS is a valuable method that is being used to independently probe attentional functions of each area within the network.

\section{Major recent advances Parietal cortex studies}

Recent TMS studies of posterior parietal cortex (PPC) have focused on two themes: PPC contributions to the processing of salient stimuli leading to the automatic allocation of attention, or 'capture', and the interaction of the two hemispheres during the voluntary spatial allocation of attention across the visual field. In general, TMS applied over PPC can either facilitate or impair attentional functions, depending on the subject's behavioral task and on the hemisphere or specific portion of PPC that is stimulated. For example, TMS to the right inferior parietal lobule prohibits the capture of attention by a salient stimulus when searching multiple-item displays (for example, a red shape among green shapes [13]). Based upon TMS applied to the right and left PPC, Mevorach et al. [14] concluded that each hemisphere processes salient stimuli differently, with the right PPC biasing attention toward salient stimuli and the left PPC biasing attention away from salient stimuli (to aid in filtering out distracters).

More strikingly still, a number of studies have found that the same TMS pulse over PPC can simultaneously enhance spatial attention in some parts of the visual field while impairing it in others [15-18]. Using a paradigm in which subjects must detect a target that appears in either the right visual field (RVF) or the left visual field (LVF) following a location cue at fixation
Figure I. Fronto-parietal attention network



Example of fronto-parietal attention network activity (in orange) projected onto the inflated cortical reconstruction of one subject. The activity of the frontal eye fields (FEFs) and posterior parietal cortex (PPC) is shown from a dorsal, or 'top', view of each hemisphere. Also shown are an example of the subject's reconstruction of the folded pial surface, which can be inflated to expose the cortex inside the sulci (above inset), and approximate positions of the traditional locations used for transcranial magnetic stimulation over the PPC (white circles), which roughly correspond to the P3 and P4 electroencephalography electrode locations over the left and right hemispheres, respectively.

[19], Thut et al. [16] showed that rTMS over the right PPC impaired target detection in the LVF but enhanced detection in the RVF when subjects were cued rightward, whereas no effects were found with leftward cues. Hung et al. [17] had subjects perform a partial report paradigm, in which locations of targets defined by a particular color had to be identified in a display containing one target, two targets, or one target and one non-target. For twotarget displays with each target placed in a hemifield, rTMS over the right PPC impaired accuracy for the left target while increasing accuracy for the right target, relative to no rTMS or left PPC rTMS. This effect disappeared when displays contained only one target. Chambers et al. [18] found that inactivation of either the right supramarginal gyrus or the right SPL enhanced the 
localization of ipsilateral (RVF) targets, but not contralateral (LVF) targets, in cluttered visual displays. This boost in spatial attention to stimuli ipsilateral to the activation site and corresponding contralateral decrement is consistent with behavioral data from patients with PPC lesions suffering from extinction, who fail to detect contralesional visual stimuli when in the presence of competing ipsilesional stimuli [20]. Together, TMS and patient studies suggest that both hemispheres are normally under mutual inhibition, and when damage or inactivation occurs in one hemisphere, the intact hemisphere is disinhibited, leading to a performance enhancement in the visual field contralateral to the intact hemisphere.

\section{Frontal cortex studies}

Traditionally, the FEF has been thought to be primarily part of the oculomotor circuit. Recent TMS studies emphasize the growing evidence that the FEF generates additional top-down signals that are not directly related to the control of oculomotor behavior. Silvanto et al. [21] used dual-site TMS stimulation of either the right or left FEF in conjunction with stimulation over the motion-selective middle temporal (MT) area to examine subjects' self reports of phosphenes. Phosphenes are brief visual sensations (light flashes) that can be induced by TMS over the visual cortex. FEF TMS significantly lowered subjects' phosphene thresholds, thereby demonstrating that the FEF directly modulates visual cortex activity in a top-down fashion. This finding suggests that, while the FEF is primarily part of an oculomotor circuit to generate and control saccadic eye movements, it may also play a more general role in top-down attentional control [22]. Several recent studies of TMS over the FEF have examined the interaction between saccadic eye movements and visuospatial attention [23,24]. Neggers et al. [23] asked subjects to perform a target discrimination task that involved saccadic eye movements either to a previously cued location or to a new location adjacent to the cued location. Saccades to targets that appeared in the previously cued location were much faster than saccades to new locations, which demonstrated a coupling between the locus of spatial attention and the prepared saccade. TMS of both right and left FEFs decreased this advantage, suggesting that saccade preparation in the FEF affects visual attention processing.

\section{Concurrent studies}

One potentially fruitful direction of study is the use of TMS in combination with other imaging and physiological methods commonly used in cognitive neuroscience, such as fMRI or EEG. Although concurrent uses of TMS/fMRI and TMS/EEG were first successfully reported more than a decade ago $[1,25]$, the simultaneous use of these methods is expensive, technically challenging, and therefore still fairly rare. However, concurrent TMS/fMRI is a powerful approach since it allows the causal effects of the pulse to be observed throughout the brain. Two recent studies using concurrent rTMS/fMRI examined the effects of disrupting the right FEF [26] and right IPS [27] on fMRI activity in visual cortex. TMS over IPS led to a decrease of activity in dorsal visual area MT as subjects passively viewed motion stimuli, but TMS over the FEF did not lead to any changes in this area. In contrast, effects on early visual cortex and ventral area V4 occurred with either IPS or FEF stimulation. Taken together, these studies suggest that the IPS and FEF have functionally distinct roles within the fronto-parietal network. These studies also demonstrate that TMS is able to induce widespread cortical activation, suggesting that TMS can affect longranging projection fibers. These findings question the spatial specificity of TMS since the actual source of an observed behavioral change could be located downstream from the inactivated region. However, further studies are needed to determine whether TMS has a functional impact on these distant regions of activation.

TMS and EEG can be used in combination to examine the fine temporal dynamics of cognitive processing throughout the brain since both methods have fairly high temporal resolution. While subjects performed an attention-orienting task that involved detecting a target that appeared in either the RVF or LVF following a location cue at fixation, Taylor et al. [28] used rTMS to disrupt the right FEF and recorded EEG activity over right and left visual cortices immediately after delivery of each pulse. TMS over the right FEF caused a sustained negative deflection of ipsilateral (right hemisphere, or RH) electrodes over visual cortex but no effects in contralateral (left hemisphere, or LH) electrodes. The authors concluded that FEF TMS had a causal impact on visual cortical activity, which is consistent with neuroimaging and physiological evidence that fronto-parietal areas are likely sources of attention-related modulatory signals in the visual cortex $[29,30]$. In agreement with these results, a recent TMS/EEG study found that FEF stimulation induced visual cortex activation in a feature-specific manner [31].

\section{Future directions}

Although previous TMS studies have begun to provide some insight into the roles that the FEF and PPC contribute to attentional control, future studies should further investigate the functional differences between these two brain regions. Some investigators distinguish the two brain regions based on the idea that the FEF is primarily a motor output area whereas the PPC is primarily a sensory area, but this distinction is not so 
straightforward. Both the FEF and PPC contain saccadic movement neurons, visually responsive neurons, and neurons that exhibit elevated delay activity (that is indicative of a working memory trace). A recent set of reversible inactivation studies found that, when injected into the lateral intraparietal area [32] and FEF [33], muscimol, a GABA (gamma-aminobutyric acid) agonist, greatly impaired a monkey's ability to detect a stimulus in the contralateral visual field. In addition, these two regions are nearly always activated together in covert visuospatial attention studies using fMRI and are extensively connected to one another anatomically. Despite these similarities, these two areas presumably contribute to attentional control in subtly different ways. TMS is in a unique position to tease apart the functions of each, perhaps through the use of dual-site methods.

There is also the possibility that different portions of PPC play different roles in attentional control. Many of the current studies continue to determine PPC location using scalp positions $\mathrm{P} 3$ and $\mathrm{P} 4$ from the international standardized 10/20 EEG electrode system, but the spatial imprecision of this method of localization does not account for inter-subject anatomical variation and the diversity in function of PPC subregions. Some of the diverse findings from TMS studies of the PPC may be due to the fact that PPC regions with different functionality were jointly inactivated. An increasing number of studies have begun to use fMRI-based frameless stereotaxy to help guide coil placement. Frameless stereotaxy uses external tracking devices and an infrared positioning system to align a subject's head in three-dimensional space to a corresponding MRI image and fMRI activation maps in virtual space. This technique may result in greater precision when inactivating distinct regions within a network.

Another avenue for future research involves the functional differences between the PPC and FEF in the RH and LH. Several of the studies reviewed here have reported altered attention functions with TMS over the RH but have failed to find changes after left PPC or left FEF disruption $[13,17,21]$. These findings are consistent with the idea that there may be some $\mathrm{RH}$ predominance for attentional control, as suggested from behavioral studies performed with patients suffering from visuospatial hemineglect $[34,35]$. Visuospatial hemineglect is a disorder caused by circumscribed lesions of frontal cortex and/or PPC, most often to the RH, leading to a deficit in attention to and awareness of one or multiple reference frames, most often affecting the contralateral (left) side of space or an object. Due to results from the patient literature, many recent TMS studies have chosen not to probe FEF or PPC function in the LH $[16,27,28]$.
However, many fMRI studies show bilateral activation of fronto-parietal regions during covert spatial attention, suggesting that LH regions are involved. More TMS studies over left regions of the attention network are needed to determine the contributions of the $\mathrm{LH}$ to attentional control.

TMS could also be used to systematically study the existence of attentional asymmetries in the non-human primate brain. Asymmetries within the human attention system (and other cognitive domains) are well documented [35]. However, based on evidence from singlecell studies, no such differences appear to exist between hemispheres in the macaque brain. TMS studies in awake, behaving monkeys may provide an avenue for testing possible hemispheric asymmetries of cognitive functions in the macaque brain. The use of TMS in the non-human primate may have an advantage over pharmacological inactivation techniques, such as the use of muscimol, because of TMS's superior temporal resolution, and also over single-cell physiology because of TMS's ability to simultaneously probe an entire network. In summary, TMS provides a valuable complement to correlative methods and will help provide a more complete picture of the functional roles of frontoparietal cortex in the control of visuospatial attention.

\section{Abbreviations}

EEG, electroencephalography; FEF, frontal eye field; fMRI, functional magnetic resonance imaging; GABA, gamma-aminobutyric acid; IPS, intraparietal sulcus; LH, left hemisphere; LVF, left visual field; MT, middle temporal; PPC, posterior parietal cortex; $\mathrm{RH}$, right hemisphere; RVF, right visual field; rTMS, repetitivepulse transcranial magnetic stimulation; SEF, supplementary eye field; SPL, superior parietal lobule; TMS, transcranial magnetic stimulation.

\section{Competing interests}

The authors declare that they have no competing interests.

\section{Acknowledgments}

This work was supported by a National Institutes of Health grant (RO1 MH64043).

\section{References}

I. Ilmoniemi RJ, Virtanen J, Ruohonen J, Karhu J, Aronen HJ, Naatanen R, Katila T: Neuronal responses to magnetic stimulation reveal cortical reactivity and connectivity. Neuroreport 1997, 8:3537-40.

2. Thut G, Northoff G, Ives JR, Kamitani Y, Pfennig A, Kampmann F, Schomer DL, Pascual-Leone A: Effects of single-pulse transcranial magnetic stimulation (TMS) on functional brain activity: a combined event-related TMS and evoked potential study. Clin Neurophysiol 2003, I | 4:207|-80. 
3. Epstein CM, Schwartzberg DG, Davey KR, Sudderth DB: Localizing the site of magnetic brain stimulation in humans. Neurology 1990, 40:666-70.

4. Lang N, Harms J, Weyh T, Lemon RN, Paulus W, Rothwell JC, Siebner HR: Stimulus intensity and coil characteristics influence the efficacy of rTMS to suppress cortical excitability. Clin Neurophysiol 2006, I I 7:2292-30I.

5. Baker SN, Olivier E, Lemon RN: Recording an identified pyramidal volley evoked by transcranial magnetic stimulation in a conscious macaque monkey. Exp Brain Res 1994, 99:529-32.

6. Baker SN, Olivier E, Lemon RN: Task-related variation in corticospinal output evoked by transcranial magnetic stimulation in the macaque monkey. J Physiol 1995, 488:795-80I.

7. Di Lazzaro V, Oliviero A, Pilato F, Mazzone P, Insola A, Ranieri F, Tonali PA: Corticospinal volleys evoked by transcranial stimulation of the brain in conscious humans. Neurol Res 2003, 25: | 43-50.

8. Moliadze $\mathrm{V}$, Zhao $\mathrm{Y}$, Eysel U, Funke K: Effect of transcranial magnetic stimulation on single-unit activity in the cat primary visual cortex. J Physiol 2003, 553:665-79.

9. Daskalakis ZJ, Levinson AJ, Fitzgerald PB: Repetitive transcranial magnetic stimulation for major depressive disorder: a review. Can J Psychiatry 2008, 53:555-66.

10. Kastner S, Ungerleider LG: Mechanisms of visual attention in the human cortex. Annu Rev Neurosci 2000, 23:3I5-4I.

II. Corbetta M, Shulman GL: Control of goal-directed and stimulusdriven attention in the brain. Nat Rev Neurosci 2002, 3:20I-I5.

12. Yantis S, Serences JT: Cortical mechanisms of space-based and object-based attentional control. Curr Opin Neurobiol 2003, 13:187-93.

13. Hodsoll J, Mevorach C, Humphreys GW: Driven to less distraction: rTMS of the right parietal cortex reduces attentional capture in visual search. Cereb Cortex 2009, 19:106-14.

14. Mevorach C, Humphreys GW, Shalev L: Opposite biases in salience-based selection for the left and right posterior parietal cortex. Nat Neurosci 2006, 9:740-2.

15. Hilgetag CC, Theoret H, Pascual-Leone A: Enhanced visual spatial attention ipsilateral to rTMS-induced 'virtual lesions' of human parietal cortex. Nat Neurosci 200I, 4:953-7.

16. Thut G, Nietzel A, Pascual-Leone A: Dorsal posterior parietal rTMS affects voluntary orienting of visuospatial attention. Cereb Cortex 2005, 15:628-38.

\section{FI000 Factor 6.0 Must Read \\ Evaluated by Marlene Behrmann 04 Nov 2004}

17. Hung J, Driver J, Walsh V: Visual selection and posterior parietal cortex: effects of repetitive transcranial magnetic stimulation on partial report analyzed by Bundesen's theory of visual attention. J Neurosci 2005, 25:9602-12.

18. Chambers CD, Stokes MG, Janko NE, Mattingley JB: Enhancement of visual selection during transient disruption of parietal cortex. Brain Res 2006, 1097:149-55.

19. Posner MI, Snyder CR, Davidson BJ: Attention and the detection of signals. J Exp Psychol 1980, 109:160-74.

20. Bisiach $E$, Vallar G: Unilateral neglect in humans. In Handbook of Neuropsychology. Vol. I, 2nd edition. Edited by Boller F, Grafman J, Rizzolatti G: Oxford, UK: Elsevier; 2000:459-502.
2I. Silvanto J, Lavie N, Walsh V: Stimulation of the human frontal eye fields modulates sensitivity of extrastriate visual cortex. J Neurophysiol 2006, 96:94I-5.

22. Schall JD: Neural basis of saccade target selection. Rev Neurosci 1995, 6:63-85.

23. Neggers SF, Huijbers W, Vrijlandt CM, Vlaskamp BN, Schutter DJ, Kenemans JL: TMS pulses on the frontal eye fields break coupling between visuospatial attention and eye movements. J Neurophysiol 2007, 98:2765-78.

24. Juan $\mathrm{CH}$, Muggleton NG, Tzeng OJ, Hung DL, Cowey A, Walsh V: Segregation of visual selection and saccades in human frontal eye fields. Cereb Cortex 2008, 18:2410-5.

25. Bohning DE, Pecheny AP, Epstein CM, Speer AM, Vincent DJ, Dannels W, George MS: Mapping transcranial magnetic stimulation (TMS) fields in vivo with MRI. Neuroreport 1997, 8:2535-8.

26. Ruff CC, Blankenburg F, Bjoertomt O, Bestmann S, Freeman E, Haynes JD, Rees G, Josephs O, Deichmann R, Driver J: Concurrent TMS-fMRI and psychophysics reveal frontal influences on human retinotopic visual cortex. Curr Biol 2006, 16:1479-88.

27. Ruff CC, Bestmann S, Blankenburg F, Bjoertomt O, Josephs $O$, Weiskopf N, Deichmann R, Driver J: Distinct causal influences of parietal versus frontal areas on human visual cortex: evidence from concurrent TMS-fMRI. Cereb Cortex 2008, 18:817-27.

FI000 Factor 3.0 Recommended Evaluated by Mark D'Esposito 07 Nov 2007

28. Taylor PC, Nobre AC, Rushworth MF: FEF TMS affects visual cortical activity. Cereb Cortex 2007, I7:39I-9.

29. Kastner S, Pinsk MA, De Weerd P, Desimone R, Ungerleider LG: Increased activity in human visual cortex during directed attention in the absence of visual stimulation. Neuron 1999, 22:75I-6I.

30. Moore T, Armstrong KM: Selective gating of visual signals by microstimulation of frontal cortex. Nature 2003, 421:370-3.

FI000 Factor 8.2 Exceptional Evaluated by Hidehiko Komatsu 07 Feb 2003, David Heeger 31 Jul 2003, John Reynolds I4 Nov 2003

3I. Morishima Y, Akaishi R, Yamada Y, Okuda J, Toma K, Sakai K: Taskspecific signal transmission from prefrontal cortex in visual selective attention. Nat Neurosci 2009, 12:85-91.

32. Wardak C, Olivier E, Duhamel JR: A deficit in covert attention after parietal cortex inactivation in the monkey. Neuron 2004, 42:50I-8

33. Wardak C, Ibos G, Duhamel JR, Olivier E: Contribution of the monkey frontal eye field to covert visual attention. J Neurosci 2006, 26:4228-35.

34. Heilman KM, Van Den Abell T: Right hemisphere dominance for attention: the mechanism underlying hemispheric asymmetries of inattention (neglect). Neurology 1980, 30:327-30.

35. Mesulam MM: Spatial attention and neglect: parietal, frontal and cingulate contributions to the mental representation and attentional targeting of salient extrapersonal events. Philos Trans R Soc Lond B Biol Sci 1999, 354:1325-46. 